\title{
The line-of-sight proximity effect in individual quasar spectra ${ }^{\star}, \star \star$
}

\author{
A. Dall'Aglio, L. Wisotzki, and G. Worseck
}

\author{
Astrophysikalisches Institut Potsdam, An der Sternwarte 16, 14482 Potsdam, Germany \\ e-mail: adaglio@aip.de
}

Received 11 January 2007 / Accepted 7 January 2008

ABSTRACT

\begin{abstract}
We exploit a set of high signal-to-noise $(\sim 70)$, low-resolution $(R \sim 800)$ quasar spectra to search for the signature of the so-called proximity effect in the $\mathrm{HI}_{\mathrm{I}} \mathrm{Ly} \alpha$ forest. Our sample consists of 17 bright quasars in the redshift range $2.7<z<4.1$. Analysing the spectra with the flux transmission technique, we detect the proximity effect in the sample at high significance. We use this to estimate the average intensity of the metagalactic UV background, assuming it to be constant over this redshift range. We obtain a value of $J=(9 \pm 4) \times 10^{-22} \mathrm{erg} \mathrm{cm}^{-2} \mathrm{~s}^{-1} \mathrm{~Hz}^{-1} \mathrm{sr}^{-1}$, in good agreement with previous measurements at similar $z$. We then apply the same procedure to individual lines of sight, finding a significant deficit in the effective optical depth close to the emission redshift in every single object except one (which by a different line of evidence does nevertheless show a noticeable proximity effect). Thus, we clearly see the proximity effect as a universal phenomenon associated with individual quasars. Using extensive Monte-Carlo simulations to quantify the error budget, we assess the expected statistical scatter in the strength of the proximity effect due to shot noise (cosmic variance). The observed scatter is larger than the predicted one, so that additional sources of scatter are required. We rule out a dispersion of spectral slopes as a significant contributor. Possible effects are long time-scale variability of the quasars and/or gravitational clustering of Ly $\alpha$ forest lines. We speculate on the possibility of using the proximity effect as a tool to constrain individual quasar ages, finding that ages between $\sim 10^{6}$ and $\sim 10^{8}$ yrs might produce a characteristic signature in the optical depth profile towards the QSO. We identify one possible candidate for this effect in our sample.
\end{abstract}

Key words. cosmology: diffuse radiation - galaxies: intergalactic medium - galaxies: quasars: absorption lines

\section{Introduction}

The multitude of absorption lines seen in the spectra of high redshift quasars gives important information about the state of matter in the universe, tracing the physical conditions of the intergalactic medium (IGM) at various epochs. It is commonly believed that for column densities up to $N_{\mathrm{HI}} \approx 10^{17.5} \mathrm{~cm}^{-2}$, the absorbers are in photoionisation equilibrium with a metagalactic ultraviolet background field (UVB), composed of the integral over all sources of UV radiation (essentially, star-forming galaxies and quasars). High-resolution spectra of the Lyman forest provide not only a rather detailed statistical characterisation of the absorber properties such as line number densities as well as temperature and density distributions (e.g., Kim et al. 2001), but also physical parameters such as H I and He II photoionisation rates (Rauch et al. 1997; Fardal et al. 1998), which directly relate to the intensity of the UVB, as described recently by Bolton et al. (2005) invoking hydrodynamical simulations. Independently, the UVB has been successfully synthesised by combining the observed quasar luminosity function and the UV emission from galaxies (although the latter is still very uncertain) with the propagation of diffuse radiation (Haardt \& Madau 1996).

In the vicinity of strong UV sources such as bright quasars, the H I photoionisation rate should locally increase, further reducing the density of residual neutral hydrogen. This enhances the transparency of the IGM to H I ionising radiation and should

\footnotetext{
* Based on observations collected at the European Southern Observatory, Paranal, Chile (Programme 070.A-0425).

$\star \star$ Figures A.1-A.17 are only available in electronic form at http://www . aanda.org
}

become observable as a weakening of the Lyman forest absorption near such sources. Such an effect has first been noted by Carswell et al. (1982) and was later baptised "Inverse" (Murdoch et al. 1986) or "Proximity Effect" Bajtlik et al. (1988, hereafter BDO88). Its prime application has been so far the possibility to derive an independent estimate of the UVB intensity, by measuring the reduction of column densities (against the global evolution of absorption line density increasing with redshift) and combining this with the QSO luminosity at the Lyman limit, assumed to be known. The best constraints on the UVB using the proximity effect stem from the combined analysis of large quasar samples (Cooke et al. 1997; Scott et al. 2000; Liske \& Williger 2001), yielding mostly values consistent with the above quoted other methods. However, the uncertainties are still substantial. Besides the problem that a limited number of lines of sight always suffers from "cosmic variance", there may also be systematic biases. In particular, if QSOs reside in intrinsically overdense environments then the signature of the proximity effect will appear weaker than predicted (Loeb \& Eisenstein 1995; Rollinde et al. 2005). Another uncertainty is the possibly limited lifetime of quasars. On the other hand, the proximity effect may also be used to derive constraints on this important, but largely unknown astrophysical quantity (e.g., Pentericci et al. 2002).

In this paper we present an exploitation of new observational material in terms of the proximity effect (Sect. 2). Rather than the traditional line counting we use the more sensitive flux transmission statistic to search for proximity effect signatures, augmented by extensive Monte-Carlo simulations to calibrate the systematic and statistical errors (Sect. 3). We deliver our results in Sects. 4.1 and 5. Firstly we briefly present an analysis of the combined sample of 17 QSO spectra and derive an estimate of 


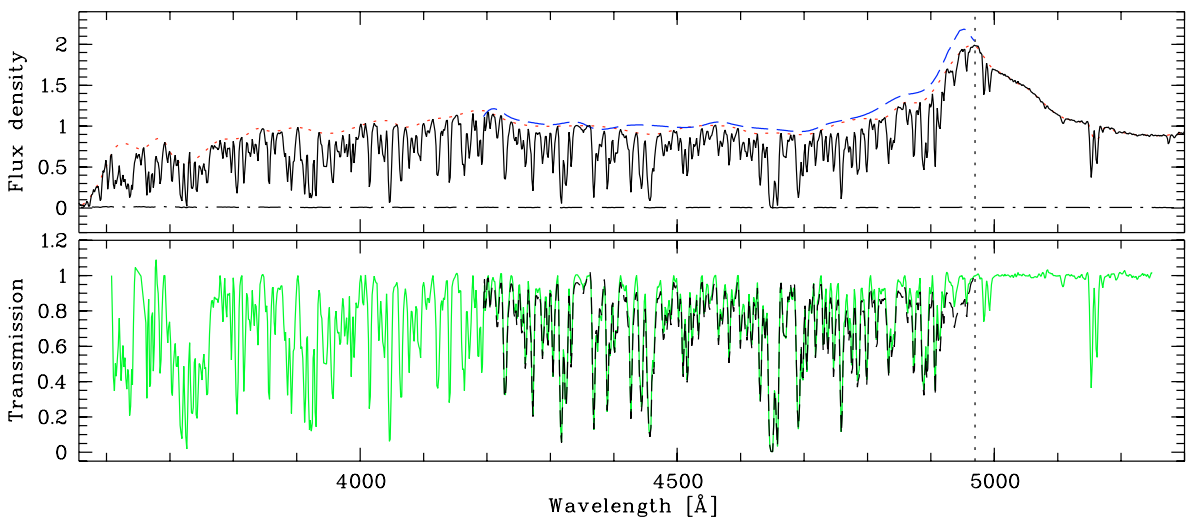

Fig. 1. Example of a quasar spectrum taken from the dataset (HE 0940-1050, $z=3.088$ ). In the upper panel the flux density in units of erg cm ${ }^{-2} \mathrm{~s}^{-1} \AA^{-1}$ as function of wavelength is presented with the uncorrected (dotted line), corrected (long-dashed line) continuum level and the noise of the spectrum (dashed-dotted line). In the lower panel the fitted transmission is shown in gray while the corrected continuum is plotted in black (See Sects. 2.1 and 3.2.3 for details). In both panels the vertical dotted line represent the emission redshift. the UV background intensity. Secondly we demonstrate that the effect is measurable on single sightlines (Williger et al. 1994; Lu et al. 1996; Savaglio et al. 1997), not only statistically in large samples (Bajtlik et al. 1988; Scott et al. 2000; Liske \& Williger 2001).

The effect can actually be systematically detected in each single QSO of our sample (with one special case that is discussed separately). We speculate about the possibilities to detect signatures of finite quasar ages by virtue of the proximity effect.

Throughout this paper, we assume a flat $\Lambda$-Universe with $\Omega_{\mathrm{m}}=0.3$ and $\Omega_{\Lambda}=0.7$ and $H_{0}=71 \mathrm{~km} \mathrm{~s}^{-1} \mathrm{Mpc}^{-1}$.

\section{Data}

\subsection{Observations and data reduction}

Our data were obtained at the ESO-VLT UT4 (Yepun) in service mode between Oct. 2002 and Apr. 2003. We used FORS2 in long slit spectroscopy mode with the 600B grism, covering the range 3315-6360 A. A longitudinal atmospheric dispersion corrector (LADC) was used to account for differential refraction effects. With a slit width of $1^{\prime \prime}$, the resolution is $\sim 800$ (4 $\AA$ FWHM). Table 1 summarises the observations for the full sample. In total we observed 17 QSOs, always fully covering the Ly Forest spectrum between the $\operatorname{Ly} \alpha$ and $\operatorname{Ly} \beta$ emission lines.

The spectra were reduced using IRAF standard procedures. Each exposure was bias-corrected and flat-fielded, cosmic rays were marked using a $\kappa-\sigma$ clipping algorithm, and the images were finally sky-subtracted. The extracted spectra were calibrated in wavelength and flux and corrected for vacuum and heliocentric shifts. The galactic extinction was taken into account assuming the $E(B-V)$ estimations by Schlegel et al. (1998), together with the Cardelli et al. (1989) extinction curve assuming $R_{\mathrm{V}}=3.1$. The two exposures available for each target were coadded with inverse variance weights, yielding a typical signalto-noise ratio $(S / N)$ of $\sim 70$ in the Lyman forest.

\subsection{Quasar magnitudes}

An evaluation of the proximity effect relies on the accurate knowledge of quasar fluxes. Even though absolute spectrophotometry is compromised by intrinsic quasar variability, we reached reasonable accuracy in almost all spectra (see Table 1 for details). We accounted for slit losses modelling a Gaussian point spread function with FWHM given by the average seeing during the observations. Centering the 1 arcsec slit on the Gaussian centroid, we corrected for the flux falling outside the slit in all our spectra. In addition to our data, we used images obtained at ESO-VLT UT1 (Antu) in Nov. 2004 under photometric conditions, covering 3 of our fields and SDSS information for 4 quasars yielding consistency to within $\delta V$ mag $\leq 0.1$. For those spectra taken in clear to photometric conditions (6 in total), the corrected magnitudes matched the values from Véron-Cetty \& Véron (2006) within $\delta V$ mag $\leq 0.1$. The $V$ magnitudes of the remaining objects, after slit loss correction, were systematically lower than the Véron-Cetty \& Véron (2006) values by not more than 0.3 mag. Since those data were taken in relatively poor sky conditions, we adopted the Véron-Cetty \& Véron (2006) values and associated larger uncertainties $(\delta V$ mag $\leq 0.3)$.

\subsection{Systemic quasar redshifts}

Our spectra cover a sufficient range in wavelength so that we could measure the redshift of each quasar from more than one emission line. All measured redshifts are compiled in Table 2. In order to adopt a systemic redshift we used low-ionisation lines whenever possible (Gaskell 1982; Tytler \& Fan 1992). For objects where this was unfeasible, we used the redshift from highionisation lines with a statistical correction, determined from the average shift between $\mathrm{Si}$ II+O I and Si IV+O IV]. The agreement between $\mathrm{Si}$ II+O I and C II estimates is generally good, even though the second line is usually very weak and rather broad. For Q 0000-26 only the asymmetric Ly $\alpha$ line with strong associated absorption was covered by our spectrum; for this object we adopted the redshift from Schneider et al. (1991). For Q 0347-383 our corrected value is in agreement with the estimation done by Steidel (1990).

\subsection{Quasar continuum}

The analysis of absorption lines requires a normalisation to the QSO continuum. We explored two types of continuum estimates: (i) a global power law $\left(f(v) \propto v^{\alpha}\right)$, excluding emission and absorption regions, used to estimate the quasar flux at the Lyman limit; (ii) a more local estimate that also includes the broad emission lines as quasi-continuum. For this task we developed an automatic algorithm, following the work by Young et al. (1979) and Carswell et al. (1982), which perform a cubic spline interpolation based on adaptive intervals along the spectrum with respect to the continuum slope. The points for the spline interpolation were chosen starting from a regular sampling of the spectrum with a binning that becomes finer whenever the slope of the computed continuum exceeds a given threshold. This is done in order to better reproduce the wings of emission lines.

In Sect. 3.2.3 below we assess the expected errors (arising mainly from line crowding) associated with this process. Figure 1 shows a sample quasar spectrum together with the 
Table 1. Log of observations.

\begin{tabular}{lcccccccc}
\hline \hline QSO & $V$ mag & $z_{\mathrm{em}}$ & $\begin{array}{c}\text { Exp. time } \\
(\mathrm{s})\end{array}$ & $\begin{array}{c}\text { Seeing } \\
(\operatorname{arcsec})\end{array}$ & Airmass & Sky condition ${ }^{a}$ & Obs. date & Ref. mag \\
\hline CTQ 0247 & 17.4 & 3.025 & 750 & 1.29 & 1.07 & CL, WI & Dec. 8, 2002 & 4 \\
CTQ 1005 & 18.4 & 3.205 & 1500 & 1.13 & 1.21 & TN & Jan. 9, 2003 & 3 \\
CTQ 0460 & 17.5 & 3.139 & 900 & 1.45 & 1.10 & PH & Dec. 23, 2002 & 4 \\
H 0055-2659 & 17.5 & 3.665 & 600 & 1.34 & 1.02 & CL, WI & Dec. 8, 2002 & 4 \\
HE 0940-1050 & 16.4 & 3.086 & 600 & 0.86 & 1.12 & TN, TK & Nov. 26, 2002 & 3 \\
HE 2243-6031 & 16.4 & 3.010 & 600 & 1.02 & 1.24 & CL, PH & Nov. 9, 2002 & 4 \\
HE 2347-4342 & 16.7 & 2.885 & 600 & 0.77 & 1.06 & PH & Nov. 10, 2002 & 1 \\
PKS 2126-15 & 17.0 & 3.285 & 600 & 0.97 & 1.20 & PH & Oct. 29, 2002 & 4 \\
Q 0000-26 & 18.0 & 4.098 & 600 & 1.31 & 1.03 & TN, CL & Nov. 7, 2002 & 4 \\
Q 0002-422 & 17.2 & 2.767 & 600 & 1.39 & 1.45 & TK, TN & Oct. 14, 2002 & 3 \\
Q 0347-383 & 17.7 & 3.220 & 800 & 1.45 & 1.15 & TN & Jan. 9, 2003 & 1 \\
Q 0420-388 & 16.9 & 3.120 & 600 & 1.37 & 1.06 & CL, WI & Dec. 8, 2002 & 1 \\
Q 0913+0715 & 17.8 & 2.787 & 800 & 1.45 & 1.18 & TN & Jan. 9, 2003 & 2 \\
Q 1151+0651 & 18.1 & 2.758 & 900 & 0.94 & 1.17 & TN, CL & Jan. 25, 2003 & 2 \\
Q 1209+0919 & 18.5 & 3.291 & 1500 & 0.78 & 1.80 & CL, TN, TK & Jan. 1, 2003 & 2 \\
Q 1223+1753 & 18.1 & 2.945 & 900 & 0.66 & 1.35 & TN, CL & Jan. 25, 2003 & 2 \\
Q 2139-4434 & 17.7 & 3.214 & 800 & 0.85 & 1.33 & TN & Apr. 30, 2003 & 3 \\
\hline \multicolumn{2}{c}{ Legend: PH-Photometric, CL-Clear, TN-Thin cirrus, TK-Thick cirrus, WI-Windy. } & & \\
1: Worseck et al. (2007): PH conditions. 2: SDSS. 3: Véron-Cetty \& Véron (2006). 4: Slit loss corrected only.
\end{tabular}

Table 2. Redshift estimates with associated errors for the quasar sample resulting from different emission lines ${ }^{\dagger}$.

\begin{tabular}{|c|c|c|c|c|c|c|c|c|}
\hline QSO & $\begin{array}{c}z_{\mathrm{Ly} \alpha} \\
\sigma_{z}=0.003\end{array}$ & $\begin{array}{c}z_{\mathrm{Si} \mathrm{II}+\mathrm{O} \text { I }}^{a} \\
\sigma_{z}=0.003\end{array}$ & $\begin{array}{c}z_{\mathrm{C} \text { II }} \\
\sigma_{z}=0.005\end{array}$ & $\begin{array}{l}z_{\mathrm{Si} \text { IV+OIV] }}+\mathrm{O} \\
\sigma_{z}=0.003\end{array}$ & $\begin{array}{c}z_{\mathrm{C} \text { IV }} \\
\sigma_{z}=0.003\end{array}$ & $\begin{array}{c}\Delta z \\
z_{\text {Si II+O I }}-z_{\text {Si IV+O IV }]}\end{array}$ & $z_{\text {Lit }}$ & Reference \\
\hline CTQ 0247 & 3.008 & 3.025 & 3.021 & 3.016 & 3.008 & 0.009 & 3.020 & 1 \\
\hline CTQ 1005 & 3.201 & 3.205 & 3.210 & 3.196 & - & 0.009 & 3.210 & 1 \\
\hline CTQ 0460 & 3.134 & 3.139 & 3.135 & 3.128 & - & 0.011 & 3.130 & 1 \\
\hline Н $0055-2659$ & 3.650 & 3.665 & 3.659 & - & - & - & 3.655 & 2 \\
\hline HE $0940-1050$ & 3.081 & 3.086 & 3.088 & 3.074 & 3.059 & - & 3.068 & 3 \\
\hline HE $2243-6031$ & 3.004 & 3.010 & 3.008 & 3.005 & 3.004 & 0.005 & 3.010 & 4 \\
\hline HE $2347-4342$ & 2.877 & 2.885 & 2.886 & 2.870 & 2.861 & 0.015 & 2.885 & 5 \\
\hline PKS 2126-15 & 3.279 & 3.285 & 3.286 & 3.270 & - & 0.015 & 3.267 & 3 \\
\hline Q 0000-26 & 4.100 & - & - & - & - & - & 4.098 & 6 \\
\hline Q 0002-422 & 2.765 & 2.767 & 2.765 & 2.757 & 2.756 & 0.010 & 2.767 & 3 \\
\hline Q 0347-383 & 3.213 & $3.220^{b}$ & - & 3.209 & - & - & 3.222 & 12 \\
\hline Q 0420-388 & 3.117 & 3.120 & 3.120 & 3.109 & - & 0.011 & 3.110 & 2 \\
\hline Q $0913+0715$ & 2.784 & 2.787 & 2.786 & 2.779 & 2.767 & 0.008 & 2.785 & 7 \\
\hline Q $1151+0651$ & 2.755 & 2.758 & - & 2.752 & 2.754 & 0.006 & 2.762 & 8 \\
\hline Q $1209+0919$ & 3.292 & 3.291 & 3.289 & 3.278 & - & 0.013 & 3.291 & 9 \\
\hline Q $1223+1753$ & 2.935 & 2.945 & 2.944 & 2.936 & 2.930 & 0.009 & 2.936 & 10 \\
\hline Q $2139-4434$ & 3.211 & 3.214 & 3.210 & 3.197 & - & 0.017 & 3.230 & 11 \\
\hline
\end{tabular}

$\dagger$ : Wavelength used to estimate the redshifts are: Ly $\alpha=1215.67 \AA$, Si II + O I $=1305.77 \AA$, C II $=1335.30 \AA$, Si IV + O IV] = 1396.76 ̊, C IV $=1549.06 \AA$ (Morton 2003).

${ }^{a}$ Taken as systemic redshift. ${ }^{b}$ These redshifts were computed from the average shift between the redshift measurements of the Si II+O I and $\mathrm{Si}$ IV+O IV] emission lines. The average redshift shift is about 0.011 .

Ref. (1) Lopez et al. (2001), (2) Osmer et al. (1994), (3) Rollinde et al. (2005), (4) Lopez et al. (2002), (5) Reimers et al. (1997), (6) Schneider et al. (1991), (7) Pettini et al. (1997), (8) Véron-Cetty \& Véron (2006), (9) Storrie-Lombardi \& Wolfe (2000), (10) Hewett et al. (1995), (11) Hawkins \& Veron (1993), (12) Steidel (1990).

estimated local continuum and the resulting transmission spectrum (see online material for the complete set of quasar spectra).

\section{Analysis}

\subsection{The flux transmission technique}

The distribution of absorption lines along a line of sight (LOS) towards a quasar is usually expressed as a function of redshift $z$, column density $N_{\mathrm{HI}}$, and Doppler parameter $b$ in the form $\mathrm{d}^{3} n /\left(\mathrm{d} z \mathrm{~d} N_{\mathrm{HI}_{\mathrm{I}}} \mathrm{d} b\right)=\eta\left(z, N_{\mathrm{HI}}, b\right)$. Due to our limited spectral resolution we could not perform single absorber analysis. We followed instead the approach proposed by Zuo (1993) and Liske \& Williger (2001) to link the line number density to the evolution of the effective optical depth. The resulting evolution depends on redshift as

$$
\tau_{\text {eff }}=B(1+z)^{\gamma+1}
$$

where $B$ and $\gamma$ are sensitive to the resolution and the detectable column density range and the observable effective optical depth defined as the optical depth at the average transmission over a predefined wavelength interval: $\mathrm{e}^{-\tau_{\text {eff }}}=\left\langle\mathrm{e}^{-\tau}\right\rangle$. 
In order to account for local fluctuations of the ionising radiation field, we follow the approach by BDO88 which assumes intervening absorbers to be in photoionisation equilibrium with the local ionising field; furthermore an empty space, and no flux attenuation except geometric dilution. The modification introduced in the optical depth then becomes

$\tau_{\mathrm{eff}}=B(1+z)^{\gamma+1}(1+\omega)^{1-\beta}$

where $\omega$ is the ratio between the photoionisation rates of the quasar and the background and $\beta$ the slope in the column density distribution. Assuming a constant UVB in the range of redshifts of our sample, an equal spectral energy distribution of QSOs and background at $v>v_{\mathrm{LL}}$, and pure hydrogen absorbers that are isothermal, homogeneous, and randomly distributed along the LOS, we find

$\omega=\frac{f_{v}\left(\lambda_{\mathrm{LL}}\left(1+z_{\mathrm{c}}\right)\right)}{4 \pi J_{v}} \frac{1}{\left(1+z_{\mathrm{c}}\right)}\left(\frac{d_{\mathrm{L}}\left(z_{\mathrm{q}}, 0\right)}{d_{\mathrm{L}}\left(z_{\mathrm{q}}, z_{\mathrm{c}}\right)}\right)^{2}$

with $z_{\mathrm{c}}$ being the cloud redshift, $d_{\mathrm{L}}\left(z_{\mathrm{q}}, 0\right)$ the luminosity distance of the QSO as seen from the Earth, and $d_{\mathrm{L}}\left(z_{\mathrm{q}}, z_{\mathrm{c}}\right)$ as seen from the cloud. As clarified by Liske \& Williger (2001), $f_{v}\left(\lambda_{\mathrm{LL}}\left(1+z_{\mathrm{c}}\right)\right)$ is the flux at the Lyman limit which has to be weighted by a bandwidth correction. We computed then the normalised optical depth (also called $\xi$ ) which is the deviation of the detected optical depth from the one expected in the Ly Forest

$\xi=\frac{\tau_{\mathrm{eff}}}{B(1+z)^{\gamma+1}}=(1+\omega)^{1-\beta}$.

In order to quantify the reduction of $\xi$ close to a QSO we need to know the parameters $B$ and $\gamma$ quantifying the redshift evolution of the Lyman forest. These values are typically determined from high-resolution spectroscopy. Kim et al. (2002) obtained $B=0.0032$ and $\gamma=2.37$, which we used as starting values to compute the normalised optical depths for all QSOs over the full spectral range. This resulted in slightly too high average $\xi$ outside the proximity effect zones, where Eq. (1) should hold and produce a mean $\xi$ of unity. We corrected this slight mismatch by adjusting $B$ until we reached $\xi_{\omega \rightarrow 0} \sim 1$ for the Ly $\alpha$ forest region in all spectra, excluding the proximity effect zone. This resulted in final normalisation parameter of $B=0.0041$. We will quantify the impact of different normalisation strategies when presenting the results for the UV background in Sect. 4.1. Finally the slope of the column density distribution was set throughout the paper to $\beta=1.5$ (e.g. Hu et al. 1995; Kim et al. 2002), if not explicitly written.

In order to reveal the proximity effect, we now searched for a systematic departure of the normalised optical depths $\xi$ from unity for large values of $\omega$. The result for our combined sample is shown in Fig. 4, while Fig. 5 displays the run of $\xi$ versus $\omega$ for each individual QSO line of sight. Before we discuss these results we want to briefly describe our approach to quantify the statistical and systematic errors.

\subsection{Error estimates from synthetic spectra}

\subsubsection{Method}

Realistic models of the Ly forest, as already developed by Zhang et al. (1995), invoke the baryonic component in CDM simulations to trace the absorption line properties along sight lines towards QSOs. However, in a first approximation, such distributions can be considered as random processes, mathematically governed by Poisson statistics. Following this assumption we

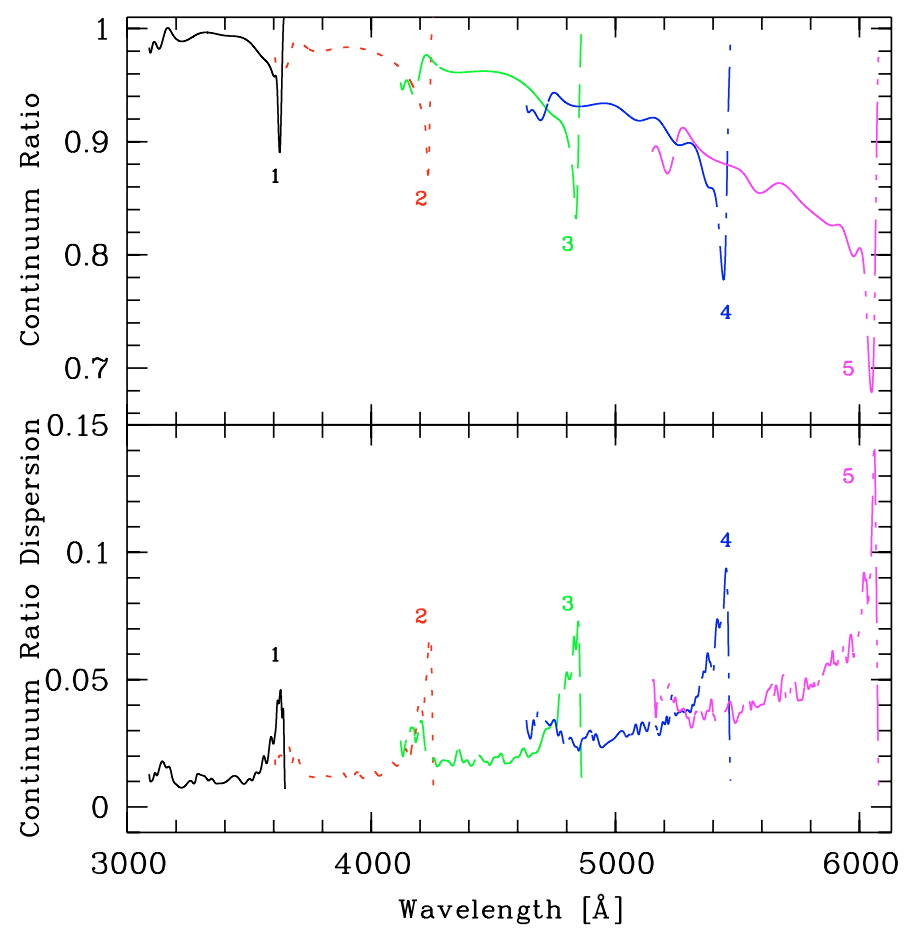

Fig. 2. Top panel: average ratio between the fitted and input continuum for the five sets of simulated QSOs. Bottom panel: standard deviation profiles relative to the above systematic bias.

performed extensive Monte-Carlo simulations to study the error budget, in particular systematic errors arising from limited spectral resolution.

Each given simulated line of sight was populated with lines distributed using $\mathrm{d} n / \mathrm{d} z \propto(1+z)^{\gamma}$ as line number density distribution leading to $\tau_{\text {eff }}=B(1+z)^{\gamma+1}$. The algorithm continues to add absorption features until the effective optical depth reaches the expected value of $\tau_{\text {eff }}$ using the best fit constraint by Kim et al. (2002) $B=0.0032, \gamma=2.37$. The column density distribution is given by $f\left(N_{\mathrm{HI}}\right) \propto N_{\mathrm{H} \text { I }}^{-\beta}$ where the slope is $\beta \sim 1.5$. The Doppler parameter distribution is given by $\mathrm{d} n / \mathrm{d} b \propto b^{-5} \exp \left[-b_{\sigma}^{4} / b^{4}\right]$ where $b_{\sigma} \simeq 24 \mathrm{~km} / \mathrm{s}$ (Kim et al. 2001) is a parameter depending on the average amplitude of the fluctuations in the velocity space of the absorbers (Hui \& Rutledge 1999). Each absorption feature was modelled as a Voigt profile and once a transmission spectrum was computed, we multiplied it by a QSO template spectrum as described in the next section.

\subsubsection{Quasar SEDs}

We generated a set of 200 artificial quasar spectral energy distributions (SEDs) via the principal component method as described by Suzuki (2006). Each rest-frame quasar spectrum can be decomposed as $f_{\lambda}(\lambda)=\mu(\lambda)+\Sigma\left(c_{i} \cdot p_{i}(\lambda)\right)$ with a mean spectrum $\mu$, the principal component spectra $p_{i}$ and the coefficients $c_{i}$. We adopted the principal components by Suzuki et al. (2005), who determined $\mu$ and $p_{i}$ at $1020 \AA<\lambda_{\mathrm{rf}}<1600 \AA$ from 50 HST FOS spectra of low-redshift quasars. The coefficients $c_{i}$ are approximately Gaussian distributed (Suzuki 2006). After generating the 200 SEDs by drawing the $c_{i}$ from their Gaussian distributions, we convolved it with the instrumental profile and added random Gaussian noise to reproduce our observations.

In order to investigate how different QSO emission redshifts affected the error budget, we simulated quasars at five typical 


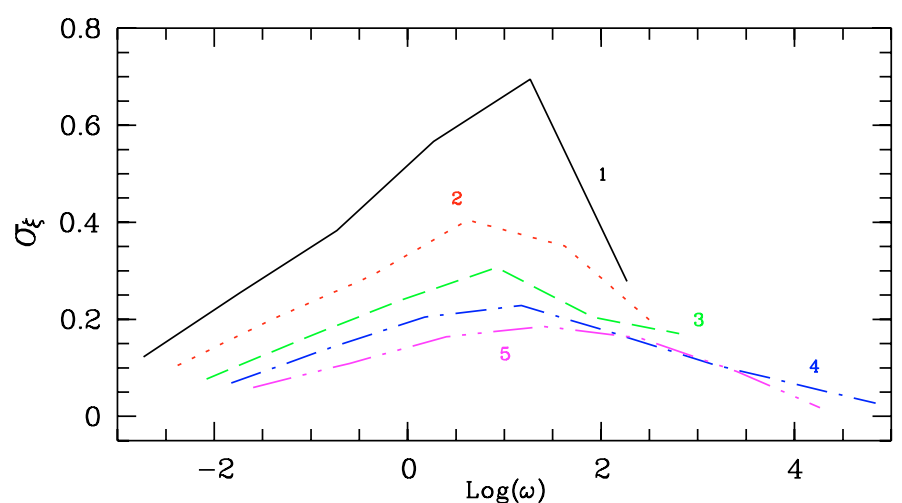

Fig. 3. Statistical (shot noise) uncertainties of the normalised optical depth close to a quasar, estimated from the Monte-Carlo simulations.

redshifts $z=2.0,2.5,3.0,3.5,4.0$, (denoted in Figs. 2, 3 with numbers 1, 2, 3, 4 and 5, respectively) and luminosities representative for our sample.

We then normalised our spectra as performed for the actual observations. These simulations created a database to quantitatively assess the two dominant sources of error: the limited number of absorbers per individual line of sight (cosmic variance), and the misplacement of the continuum because of line crowding. In the following we consider both error sources in turns.

\subsubsection{Systematic errors: continuum placement}

For each simulated spectrum we computed the ratio between the fitted and the input continuum. We then averaged the ratio over all realisations at each emission redshift. The result is shown in the top panel of Fig. 2, for the five redshifts adopted. Expectedly, the highest deviation is always in the wings of the Ly $\alpha$ emission line. It can also be seen that the increasing line number density with redshift causes a gradually growing systematic error. We use this profiles to correct our automatic continuum estimates and use the standard deviations shown in the bottom panel of Fig. 2 as contribution to the uncertainties. The effect of this correction can be seen in all the proximity effect plots as difference between the green triangles and the black dots in Figs. 4, 5 .

We note in passing that a second source of systematic errors would be the presence of metal line systems in the proximity effect zone. Our spectral resolution is insufficient to identify individual metal lines in the Lyman forest, and any such absorption present, but unaccounted for, will systematically increase the normalised optical depth $\xi$ and thus tend to mask the proximity effect.

\subsubsection{Statistical errors}

We modelled the statistical error of the measured optical depth along individual lines of sight as arising from Poissonian shot noise due to the limited number of absorbers in each simulated spectrum. For each stack of simulations we computed the mean and standard deviation of $\xi$ per $\log \omega$ bin. The results are shown in Fig. 3, which demonstrates that the standard deviations $\sigma_{\xi}$ are always considerably bigger than the continuum dispersion. However, recall that we fully account for both random and systematic errors. As expected, the statistical errors become bigger towards lower redshifts due to the smaller line number density. The simulation results were then used to describe $\sigma_{\xi}(\omega, z)$ with a simple polynomial parameterisation. Without introducing the proximity effect, the statistical error at high $\omega$ would be much larger due to cosmic variance on very small scale.

In order to estimate the statistical error for the combined analysis of the full sample we ran a new set of simulations. Here we generated 10 random lines of sight with the emission redshift of each of the 17 quasars in the sample, and computed the statistical scatter after averaging over the 17 contributions to each $\omega$ value. We found $\sigma_{\xi}$ to be essentially independent of $\omega$ in this case.

\section{The proximity effect in the combined sample}

\subsection{A new estimate of the UV background}

Figure 4 summarises the main results regarding the combined sample of 17 QSO spectra. We see a highly significant reduction of normalised optical depths at $\log \omega \gtrsim 0$, i.e. for the zone where photoionisation due to the local quasar-induced radiation field is expected to prevail over the metagalactic UV background. As already demonstrated by BDO88, this turnover can be used to constrain the mean intensity of the UVB. We adopted the fitting formula

$F(\omega)=\left(1+\frac{\omega_{-21}}{a}\right)^{1-\beta}$

with $a$ being the free parameter and $\omega_{-21}$ is the value of $\omega$ relative to a reference value of the UVB, $J_{-21}^{\star} \equiv$ $10^{-21} \mathrm{erg} \mathrm{cm}^{-2} \mathrm{~s}^{-1} \mathrm{~Hz}^{-1} \mathrm{sr}^{-1}$. We then applied a straightforward $\chi^{2}$ minimisation to search for the best-fitting value of $a=$ $J\left(v_{\mathrm{LL}}\right) / J_{-21}^{\star}$.

In doing this computation we found that the bin size $\Delta \log \omega$ has some moderate effect on the resulting best-fit value of the UVB. If the data are merged with very small or even without any binning, $a$ will be biased towards low values because of the substantial scatter in $\xi$ at very small $\omega$ (for $\log (\omega) \lessgtr-1.5$ ), due to the strong effects of shot noise for this narrow $\log (\omega)$ interval. On the other hand, too large bins will tend to hide the signature of the proximity effect, thus make the UVB appear stronger than it really is. As a reasonable compromise we chose $\Delta \log \omega=1$, upon which also Fig. 4 is based. An additional effect which tend to change the estimation of the UVB is the normalisation used to compute $\xi$ (see Eq. (4)). We address this problem with two different strategies. We use the normalisation by Kim et al. (2002) and our normalisation $(B=0.0041)$ to reach $\xi_{\omega \rightarrow 0} \sim 1$ for the combined set of Ly $\alpha$ forest regions. Table 3 summarises our results revealing a maximal dispersion of about $\sim 0.9$. We decide to use $B=0.0041$ for our results.

As best fit value we obtain $J\left(v_{\mathrm{LL}}\right)=9 \pm 4 \times$ $10^{-22} \mathrm{erg} \mathrm{cm}^{-2} \mathrm{~s}^{-1} \mathrm{~Hz}^{-1} \mathrm{sr}^{-1}$, or in logarithmic units, $\log J\left(v_{\mathrm{LL}}\right)=-21.03_{-0.22}^{+0.15}$. Using a slightly narrower bin size of $\Delta \log \omega=0.7$ lowers $J\left(v_{\mathrm{LL}}\right)$ by about $0.05 \mathrm{dex}$.

This estimate of the UVB intensity is in very good agreement with all recent measurements based on a wide range of techniques and data sets. For example, Scott et al. (2000) obtained $J\left(v_{\mathrm{LL}}\right) / J_{-21}^{\star}=0.7_{-0.44}^{+0.34}$, applying line count statistics on more than hundred spectra at $\sim 1 \AA$ resolution. More similar to our approach, Liske \& Williger (2001) used the flux transmission statistic on 10 QSO spectra with $\sim 2 \AA$ resolution and a $S / N$ of $\sim 40$, obtaining $J\left(v_{\mathrm{LL}}\right) / J_{-21}^{\star}=0.35_{-0.13}^{+0.35}$. Not much has yet been published using high resolution spectra. Giallongo et al. (1996) obtained $J\left(v_{\mathrm{LL}}\right) / J_{-21}^{\star}=0.5 \pm 0.1$ and Cooke et al. (1997) $J\left(v_{\mathrm{LL}}\right) / J_{-21}^{\star}=0.8_{-0.4}^{+0.8}$, again close to our value even though they 


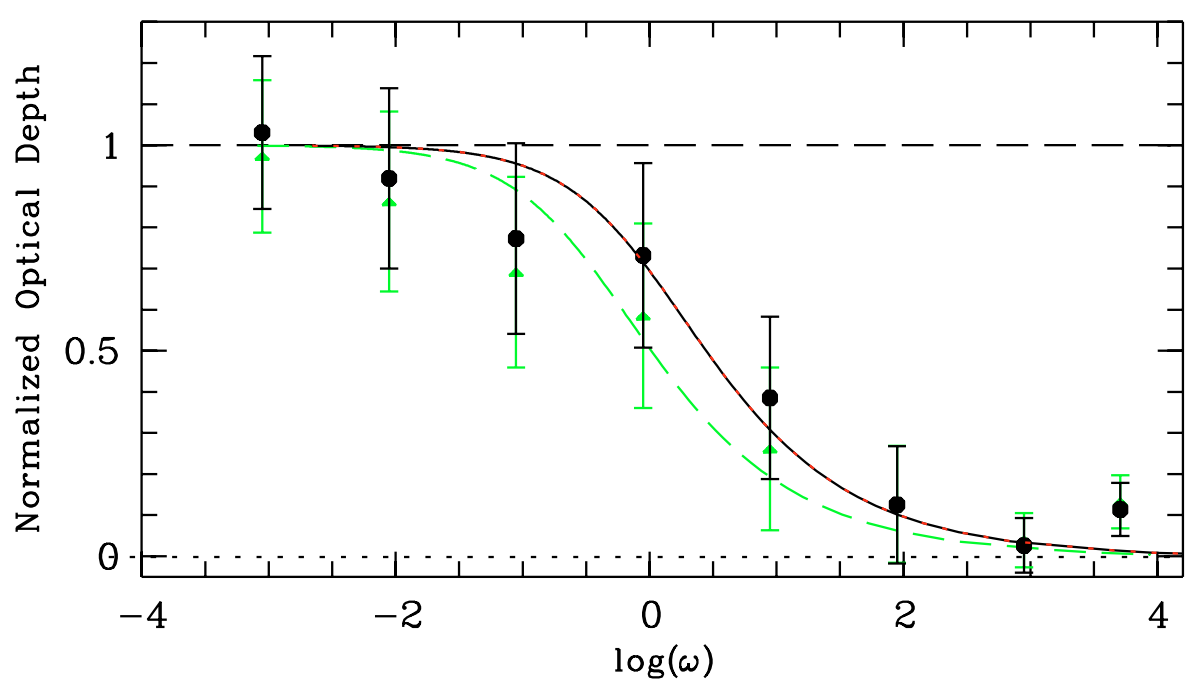

Fig. 4. Normalised optical depth versus $\omega$ profile for the combined sample of 17 quasars, binned in steps of $\Delta \log \omega=1$. The signature of the proximity effect is clearly visible for both continuum corrected and uncorrected profiles. The curved lines are the best fit of the simple photoionisation model to the data, corresponding to a UV background of $\log \left(J_{v}\right)=-21.03$ (solid line), in units of [erg cm $\mathrm{cm}^{-2} \mathrm{~s}^{-1} \mathrm{~Hz}^{-1} \mathrm{sr}^{-1}$ ], while the green long-dashed line correspond to the best fit to the continuum uncorrected data. The horizontal line refers to the case of no proximity effect.
Table 3. Estimations of the UV background $J\left(v_{\mathrm{LL}}\right) / J_{-21}^{\star}$ (computed in units of erg $\left.\mathrm{cm}^{-2} \mathrm{~s}^{-1} \mathrm{~Hz}^{-1}\right)$ from different model parameters $(\beta$ and $B$ ) and binsizes $\Delta \log \omega$.

\begin{tabular}{lllll}
\hline \hline & \multicolumn{2}{c}{$B=0.0032$} & \multicolumn{2}{c}{$B=0.0041$} \\
$\Delta \log (\omega)$ & 0.7 & 1.0 & 0.7 & 1.0 \\
\hline$\beta=1.4$ & 1.01 & 1.12 & 0.40 & 0.51 \\
$\beta=1.5$ & 1.89 & 2.04 & 0.88 & 0.93 \\
$\beta=1.6$ & 2.83 & 3.01 & 1.19 & 1.41 \\
\hline
\end{tabular}

are all smaller (up to a factor of about 2). This order of magnitude is also consistent with predictions based on the QSO luminosity function (Haardt \& Madau 1996).

\subsection{Dependence on model parameters}

Concerning the evolution of $\tau_{\text {eff }}$ in the Ly forest (see Eq. (1)), we regard only the normalisation $B$ as variable. The slope $\gamma$ has been estimated by several authors with much higher accuracy in the past years (e.g., Kim et al. 2001, 2002; Schaye et al. 2003). We consider only two normalisations: a conservative one with $B=$ 0.0032 (Kim et al. 2002), which returns a higher UVB estimate since $\xi_{\omega \rightarrow 0} \gtrsim 1$, and $B=0.0041$ which we adopt since it leads to $\xi_{\omega \rightarrow 0} \simeq 1$. We believe that the discrepancy between our adopted value and the value by Kim et al. (2002) is due to a combination of line blending and resolution effects. Our results are sensitive to the slope of the column density distribution $\beta$ which sets the steepness of $\xi(\omega)$ in the BDO88 ionisation model. The column density distribution is very well approximated by a single power law with $\beta \simeq 1.5$ over 10 dex (Petitjean et al. 1993). However, it has been shown that the slope changes somewhat with redshift (Kim et al. 2002). Since the lines are unresolved in our lowresolution spectra, we fix a single power law distribution and vary its assumed value by $\Delta \beta=0.1$ to estimate the robustness of our UVB measurements. The Doppler parameter distribution has no direct impact on our results since the BDO88 model simply assumes an isothermal $\mathrm{H}$ I distribution.

Table 3 summarises the dependence of our UVB estimates on model parameters. There is a substantial scatter in the estimations and both $B$ and $\beta$ play a central role. The binsize has only a small (but still detectable) effect.

\section{The proximity effect in individual lines of sight}

\subsection{Results}

The proximity effect is generally seen as a statistical phenomenon, which may or may not be detectable in individual spectra. The high $S / N$ of our spectra motivated us to search for proximity effect signatures in each of our 17 quasar spectra. The basic approach was essentially identical to that of the combined analysis. Compute the mean normalised optical depth for a given line of sight within a given bin $\Delta \log \omega$ and check whether $\xi$ systematically decreases for large values of $\omega$. We set the same normalisation for every object as for the combined analysis. The $\omega$ scale was now fixed by assuming the value of the mean UV background intensity from the combined analysis. The results are displayed in Fig. 5, one small panel per quasar. The error bars are now of course dominated by Poissonian shot noise, estimated from the simulations as described above. In each panel the expected run of $\xi$ with $\omega$ is shown as the dotted red line, assuming that the metagalactic UV background is constant and has the same spectral shape as the quasar. We also show the profile before the systematic continuum correction and its best fit model (green triangles and line).

Figure 5 demonstrates that in all except one case, $\xi$ decreases substantially from left to right. Thus we can say that the proximity effect is detected in 16 out of our 17 quasar spectra. In the majority of spectra, the $\xi-\omega$ profile is even formally consistent with the prediction based on the combined analysis. In a number of cases the data seem to be (mildly) discrepant with the prediction; we discuss some of these cases below.

We also applied the above fitting procedure to each spectrum separately; the results of that exercise are shown as the solid curves in Fig. 5. Table 4 summarises the fit results. Apparently the value of the fitting parameter $a$ shows significant scatter between the different quasars. The value of $a$ describes the horizontal offset of the solid curve relative to the dotted curve and in the following is regarded as a quantitative measure of the strength of the proximity effect signal (in the sense that a large $a$ means a weak proximity effect). This does of course not imply that the UV background fluctuates by a similar amount. While statistical errors certainly contribute to the scatter, one may also reinterpret the parameter $a$ as a measure of the flux of the quasar at the Lyman limit. This flux may even not always have been constant over the light travel time across the proximity effect zone; we return to that point in Sect. 5.4 below. 

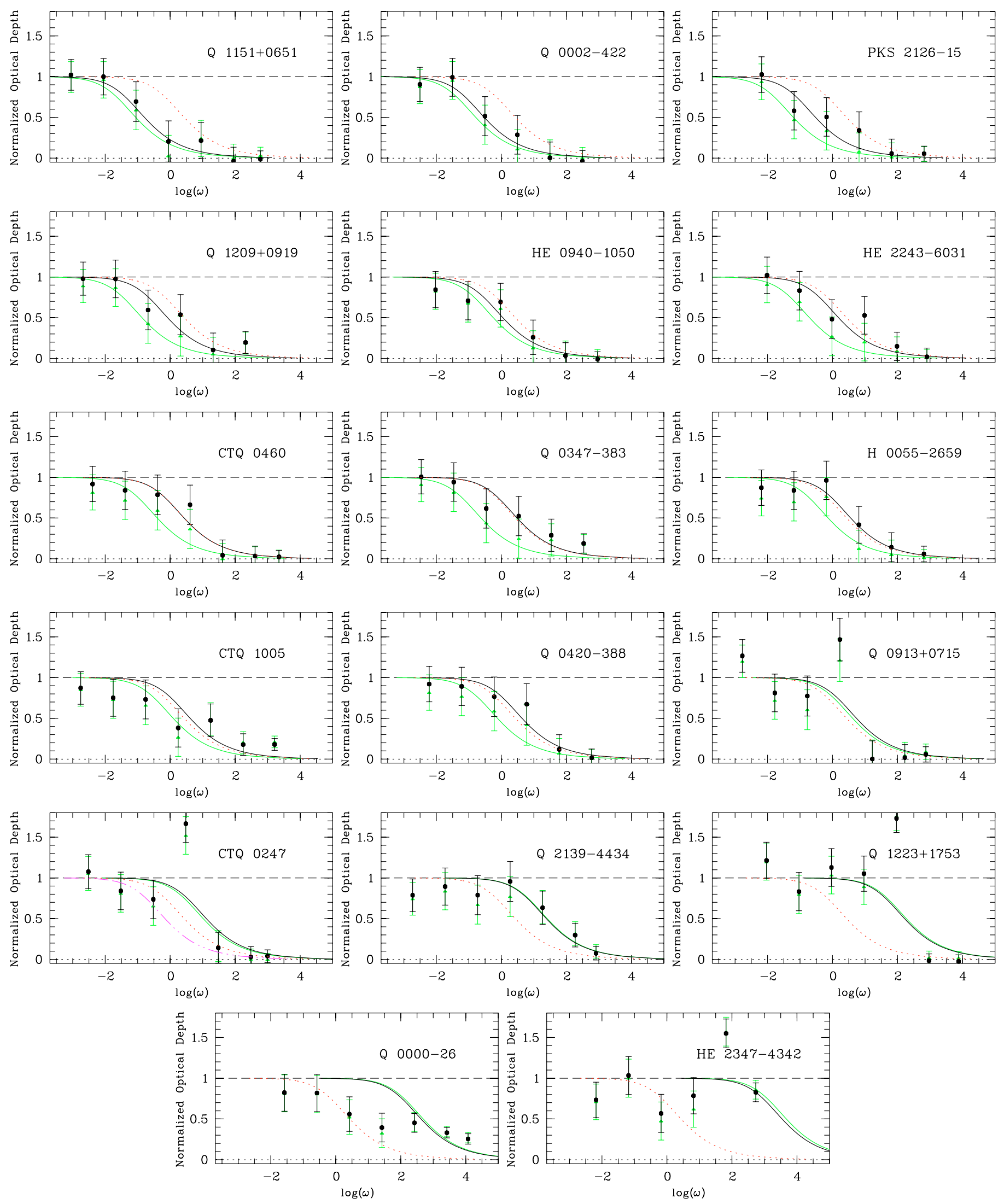

Fig. 5. Search for proximity effect signatures in individual lines of sight, for all 17 QSO spectra. Each panel shows the normalised optical depth $\xi$ versus $\omega$ in the same way as Fig. 4, with the best-fit model of the combined analysis superimposed as dotted red lines. The solid lines delineate the best fit to each individual QSO as described in the text. For CTQ 0247 we also plot the best fit excluding the strong absorption at $z \sim 3.014$ (purple curve). The panels are sorted in order of decreasing strength of the proximity effect (horizontal displacement of solid and dotted lines). HE 2347-4342 has no detectable proximity effect in H I. 
Table 4. Input and results used for the photoionisation model fits. $f_{\mathrm{LL}}$ is the predicted Lyman limit flux of each quasar. In the treatment of individual lines of sight, $\log (a)$ is the fitted parameter used to quantify the strength of the proximity effect. CTQ 0247 is listed twice, with and without the associated absorption system.

\begin{tabular}{lccc}
\hline \hline QSO & $z$ & $\log \left(f_{\text {LL }}(0)\right)$ & $\log (a)$ \\
\hline Q 1151+0651 & 2.758 & $-27.00_{-0.01}^{+0.01}$ & $-1.11_{-\infty}^{+0.51}$ \\
Q 0002-422 & 2.767 & $-26.46_{-0.05}^{+0.05}$ & $-0.84_{-\infty}^{+0.49}$ \\
PKS 2126-15 & 3.285 & $-26.42_{-0.05}^{+0.04}$ & $-0.84_{-\infty}^{+0.41}$ \\
Q 1209+0919 & 3.291 & $-26.92_{-0.01}^{+0.01}$ & $-0.40_{-\infty}^{+0.44}$ \\
HE 0940-1050 & 3.086 & $-26.16_{-0.03}^{+0.03}$ & $-0.27_{-\infty}^{+0.48}$ \\
HE 2243-6031 & 3.010 & $-26.11_{-0.02}^{+0.02}$ & $-0.14_{-\infty}^{+0.46}$ \\
CTQ 0460 & 3.139 & $-26.55_{-0.02}^{+0.03}$ & $+0.11_{-\infty}^{+0.41}$ \\
Q 0347-383 & 3.220 & $-26.67_{-0.01}^{+0.01}$ & $+0.16_{-\infty}^{+0.46}$ \\
H 0055-2659 & 3.665 & $-26.61_{-0.03}^{+0.03}$ & $+0.24_{-\infty}^{+0.43}$ \\
CTQ 1005 & 3.205 & $-26.95_{-0.03}^{+0.03}$ & $+0.29_{-\infty}^{+0.47}$ \\
Q 0420-388 & 3.120 & $-26.37_{-0.03}^{+0.03}$ & $+0.31_{-\infty}^{+0.44}$ \\
Q 0913+0715 & 2.787 & $-26.74_{-0.02}^{+0.02}$ & $+0.42_{-\infty}^{+0.50}$ \\
CTQ 0247 & 3.025 & $-26.62_{-0.03}^{+0.02}$ & $+0.70_{-\infty}^{+0.40}$ \\
CTQ 0247 & & & $-0.46_{-\infty}^{+0.49}$ \\
Q 2139-4434 & 3.214 & $-26.93_{-0.02}^{+0.01}$ & $+1.06_{-\infty}^{+0.42}$ \\
Q 1223+1753 & 2.945 & $-27.10_{-0.01}^{+0.01}$ & $+2.29_{-\infty}^{+0.44}$ \\
Q 0000-26 & 4.098 & $-26.19_{-0.05}^{+0.04}$ & $+2.30_{-\infty}^{+0.24}$ \\
HE 2347-4342 & 2.885 & $-26.20_{-0.03}^{+0.03}$ & $\gtrsim+3$ \\
\hline
\end{tabular}

We now briefly comment on two objects where the proximity effect appears to be extremely weak or absent. In the case of CTQ 0247, there is a strong associated absorption system at $z_{\mathrm{abs}}=3.014$, corresponding to $\log \omega \lesssim 1$. Removing this absorption manually from the spectrum and redoing the analysis yielded a proximity effect signal perfectly consistent with the prediction from the combined analysis.

\subsection{A hidden proximity effect for HE 2347-4342?}

For HE 2347-4342, we see no evidence at all of a downturn of $\xi(\omega)$. The absence of any proximity effect was noticed already by Reimers et al. (1997) upon mere visual inspection of the spectrum. Again there is a conspicuous strong associated absorption system. Although in this case a removal of that system does not dramatically improve the proximity effect signal, the absorber may nevertheless play an important role in this line of sight, as it may attenuate the ionising radiation field towards lower redshifts. This is supported by the following simple calculation.

The total measured H I column density in the associated system is of the order of $2 \times 10^{16} \mathrm{~cm}^{-2}$ (Fechner et al. 2004). We assume this to be located in an absorbing slab of matter very close to the QSO. Knowing the Lyman limit flux of the QSO, we can predict the value of $\omega$ at the location of the slab, $\log \omega \simeq 2.1$; this immediately relates to a predicted reduction of H I column density in the slab of $\sim 2$ dex. Thus, the column density of the same absorber without the QSO ionising radiation would be of the order of $10^{18} \mathrm{~cm}^{-2}$. This would be sufficient to render a remaining proximity effect for the line of sight undetectable.

We note that Fechner et al. (2004) detected evidence of a hard radiation field from a detailed photoionisation modelling analysis of metal absorption lines in this system. A similar trend is apparent in our recent investigation of the He II Lyman forest, combining VLT and FUSE high-resolution spectra of this quasar Worseck et al. (2007). Thus, while the traditional H I proximity

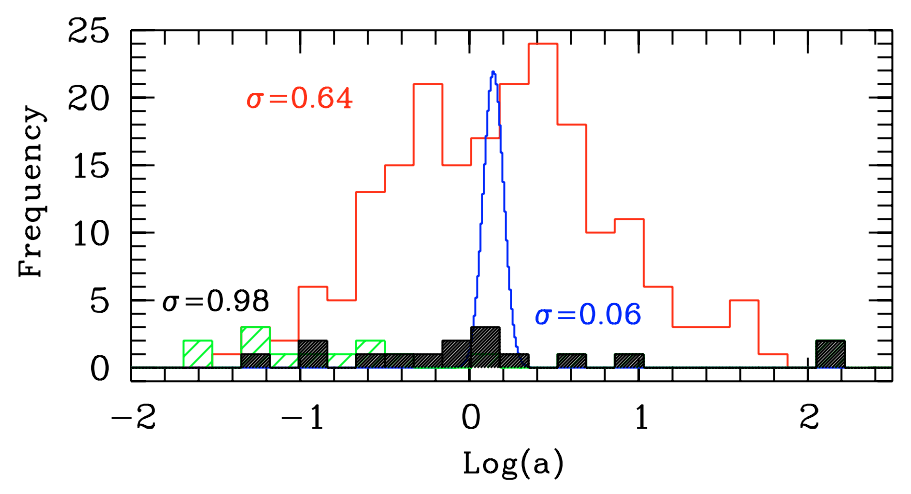

Fig. 6. Distribution of the parameter $\log (a)$ for the fits to the 17 individual spectra with and without continuum correction (black and green hashed histogram respectively). Overplotted is the distribution resulting only from shot noise in the simulated spectra (solid line), and the expected distribution of $\log (a)$ due to dispersion of spectral indices (blue Gaussian).

effect is clearly absent in HE 2347-4342, most probably due to excess absorption close to the QSO, there are clear signs of a "proximity effect in spectral hardness" (cf. Worseck \& Wisotzki 2006) for this object. We therefore conclude that all 17 quasars in our sample show evidence of a genuine proximity effect.

\subsection{Variations in the strength of the proximity effect}

We now investigate how much of the scatter in the fitting parameter $a$ might be attributed to uncertainties, or shot noise, or intrinsic dispersion of other relevant properties.

Figure 6 shows that formally the best-fit values of $a$, before and after continuum correction (green and black histograms respectively), extend over several orders of magnitudes, with a standard deviation of $\sigma_{\log (a)} \sim 1$ (black histogram).

The large dispersion can to a small part be explained by uncertainties in quasar magnitudes and redshifts, which both affect the computation of $\omega$. For those objects where we have rather accurate $V$ magnitudes (with errors $\leq 0.1 \mathrm{mag}$ ), the flux scale is accurate to within $9 \%$. For the remaining objects the flux uncertainties might be as large as $30 \%$. The direct effect on $\log (a)$ is an uncertainties of 0.03-0.1 dex. In addition, redshift errors (Table 1) might shift $\log (a)$ by $0.05-0.1$ dex. We conclude that these uncertainties cannot be the main source of spread in the estimated $\log (a)$ values.

We employed our Monte-Carlo simulations to estimate the expected scatter solely due to statistical shot noise errors (i.e., cosmic variance). To this effect we first systematically reduced the optical depths in the simulated spectra following strictly the theoretical proximity effect prescription (Eq. (4)), after which we "remeasured" the proximity effect and its strength $a$ by fitting the artificial data in the same way as the observed ones. The resulting histogram of $a$ values is superimposed in Fig. 6; the distribution is approximately Gaussian with a standard deviation of $\sigma_{\log (a)}=0.64$. This is substantial and implies that 14 our of 17 of our quasars are located within $\pm 2 \sigma$ expected for pure random errors. Nevertheless, 3 quasars from our sample are located outside of this interval, which is a bit much to declare them all as outliers.

This means that other effects play a role, and that these effects may lead to gross deviations from the simple expectation. Possible mechanisms might be, for example, a dispersion in spectral indices for the quasars, long time scale variability of 
the quasars, or a strongly fluctuating UV background. We first consider the effects of non-uniform spectral indices.

In the standard analysis of the proximity effect as introduced by BDO88, the assumption of a uniform quasar spectral index is obviously wrong, but the averaging over samples of quasars makes the analysis very insensitive to any intrinsic dispersion. When considering individual quasar lines of sight as we do here, this assumption may be more harmful. We investigated this by extending the original calculation by BDO88, solving the exact integrals for the ratio of the photoionisation rates of the quasar and the background and using the photoionisation cross section for a pure hydrogen cloud, so that

$\omega=\frac{\int_{v_{\mathrm{LL}}}^{\infty} \frac{4 \pi J_{\nu}^{\mathrm{q}}(v)}{h v} \sigma(v) \mathrm{d} v}{\int_{v_{\mathrm{LL}}}^{\infty} \frac{4 \pi J_{\nu}^{\mathrm{b}}(v)}{h v} \sigma(v) \mathrm{d} v}=\omega_{\text {old }} \frac{\kappa-3}{\alpha-3}$

where $\omega_{\text {old }}$ is the know expression of Eq. (3) and $\kappa \sim-1.8$ is the background spectral index following the model by Haardt \& Madau (1996). Expressing everything in logarithmic units we arrive at

$\log (\omega)=\log \left(\omega_{\text {old }}\right)+\sigma_{\log (a)}$.

Real quasars show a dispersion of spectral slopes of $\sigma_{\alpha}=0.25$ around $\bar{\alpha}=-0.46$ (derived from the SDSS QSO composite spectrum and its standard deviation published by Vanden Berk et al. 2001). The values of $\omega$ are therefore offset (i) by $\Delta \log (\omega)=$ 0.142 on average, and (ii) by a random component with a standard deviation of $\sigma_{\log (a)}=0.06$ (included as the narrow Gaussian in Fig. 6). Obviously, the dispersion in spectral slopes is too small, by a long way, to account for the observed distribution of $\log (a)$.

Among the two other mentioned options, intrinsic fluctuations of the UV background might also contribute, but numerical simulations suggest that substantial fluctuations are only expected for redshifts higher than those covered here (Croft et al. 1999; McDonald et al. 2005).

The presence of metal transitions falling in the proximity effect zone or a still imperfect continuum placement might also enhance the scatter.

A very plausible effect, on the other hand, would be significant QSO variability over timescales of the light travel time across the proximity effect zones which is of the order of $10^{7}$ years; there is no reason to expect that quasars always maintain their radiative output over such long periods. If they do not, then the observed Lyman limit luminosity will not be the same as that received by clouds along various points along the line of sight. We will discuss a specific aspect of this effect in the next section.

\subsection{Finite quasar age}

The simple theory of the proximity effect implicitly includes the assumption that quasars shine for an infinite time, or at any rate for much longer than the light crossing time of the proximity effect zone. This may be wrong, and we now ask specifically what would happen if a quasar is abruptly switched on within, say, less than a few Myrs before the observation. As soon as the quasar starts to radiate in the UV, an over-ionised "sphere" will start to expand around the quasar (always assuming spherical symmetry). The new equilibrium state with ionising photons from the local source and the UV background - requires at least some $\sim 10^{4}$ yrs to establish, which means that for even younger QSOs the proximity effect will be absent.

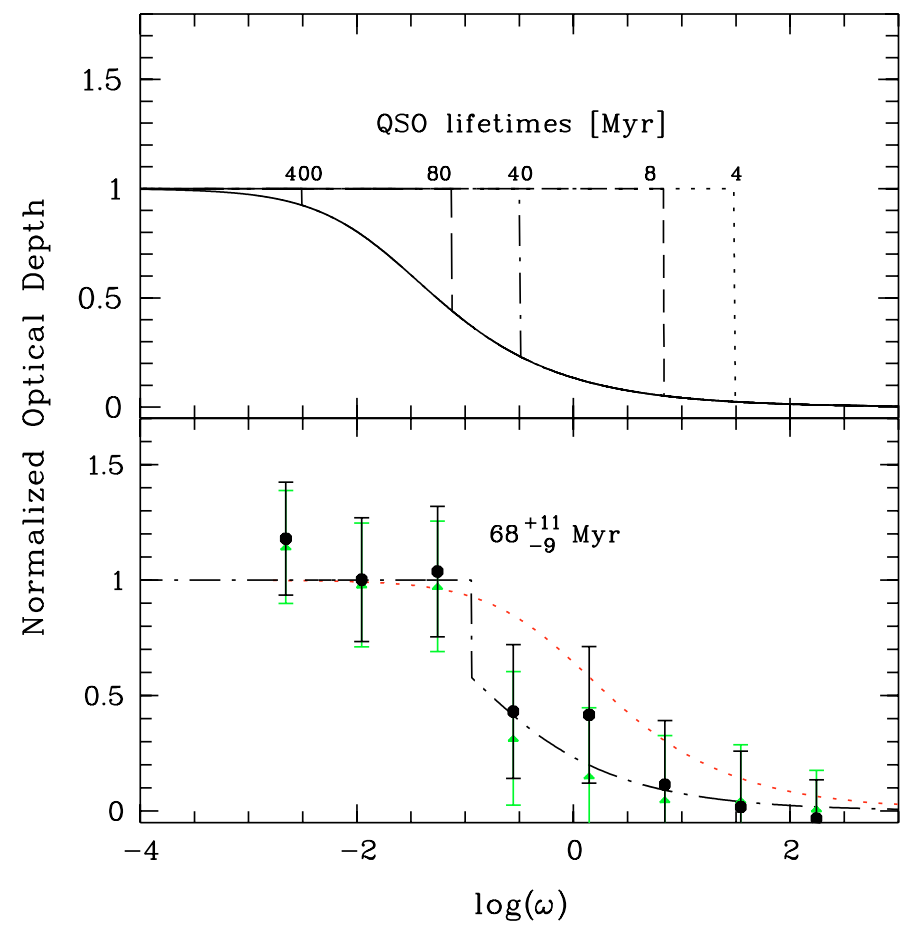

Fig. 7. Top panel: expected $\xi(\omega)$ model for different finite lifetimes of the quasar. The solid curve represents the BDO88 model of the proximity effect, with a QSO of infinite lifetime. Bottom panel: tentative application of the above model to the QSO Q 0002-422. The dotted line is the predicted $\xi(\omega)$ curve for infinite lifetime, the dot-dashed line delineates the curve for a fiducial finite lifetime of $68 \mathrm{Myr}$.

The light travel time between two redshifts along a single line of sight can be calculated for a $\Lambda$-Universe as

$t(z)=\frac{2}{3 H_{0} \sqrt{\Omega_{\Lambda}}} \operatorname{asinh} \sqrt{\frac{\Omega_{\Lambda}}{\Omega_{\mathrm{m}}(1+z)^{3}}}$

(Peacock 1999), allowing us to convert redshift intervals into light travel time differences. Switching a quasar on can be expressed in the model by introducing a step function into the $\omega$ profile,

$\omega(z)= \begin{cases}0 & \text { for } z<z_{\text {life }} \\ \frac{f_{v}\left(\lambda_{\mathrm{LL}}\left(1+z_{\mathrm{c}}\right)\right)}{4 \pi J_{v}} \frac{1}{\left(1+z_{\mathrm{c}}\right)}\left(\frac{d_{\mathrm{L}}\left(z_{\mathrm{q}}, 0\right)}{d_{\mathrm{L}}\left(z_{\mathrm{q}}, z_{\mathrm{c}}\right)}\right)^{2} & \text { for } z_{\text {life }}<z<z_{\mathrm{em}} .\end{cases}$

Far away from the QSO (low $\omega$ values), the QSO has no effect yet. When passing into the over-ionised region, the theoretical profile $\xi(\omega)$ drops abruptly, joining the BDO88 prescription of the proximity effect. This is shown in the top panel of Fig. 7, where we present the expected profiles for different assumed ages. For presentation purposes, this model was computed for a QSO at $z_{\mathrm{q}}=2.76$ and $f_{v}\left(\lambda_{\mathrm{LL}}\left(1+z_{\mathrm{q}}\right)\right)=3.46 \times$ $10^{-27} \mathrm{erg} \mathrm{cm}^{-2} \mathrm{~s}^{-1} \mathrm{~Hz}^{-1}$.

It turns out that observable signatures can be expected for an interesting range of ages. Up to $t_{\mathrm{QSO}} \sim 4 \mathrm{Myr}$ the turnover happens so close to the quasar that even for a very luminous QSO it will be hard to detect, given the inevitable shot noise limitations. Above of at most $\sim 500 \mathrm{Myr}$, on the other hand, the differences between the models with and without lifetime will wash out completely because $\xi(\omega)$ is expected to be close to unity anyway. The range between these extremes, $4 \mathrm{Myr} \lesssim t_{\mathrm{QSO}} \lesssim 400 \mathrm{Myr}$, is interestingly close to quasar lifetimes estimated by other, usually 
much more indirect methods, such as modelling of QSO accretion (Yu \& Tremaine 2002; Hopkins et al. 2006) or analysis of QSO clustering (Croom et al. 2005). It might thus be possible to detect age effects of quasars by studying their proximity effect signature.

We searched our quasars for possible examples of such a step feature, and found one (highly tentative) possible example: Q 0002-422, shown in the bottom panel of Fig. 7, seems to match the expected pattern for a "recently born quasar" quite well. The normalised optical depth $\xi$ remains constant at $\sim$ unity over the Lyman forest until $\log (\omega)=-1.5$ is reached, where it rather abruptly drops to much lower values. We chose a smaller binning in $\log \omega$ for this plot, compared to the previous figures, in order to highlight the relatively sudden drop. Applying our simple model we obtain an age of $t_{\mathrm{QSO}} \simeq 68_{-9}^{+11} \mathrm{Myr}$, where the errors are based on simply assuming the uncertainty to be \pm 0.35 bin in $\log \omega$. Of course we do not claim to have measured the age of that quasar with $20 \%$ accuracy. The difference in the goodness-of-fit between finite and infinite quasar ages is not formally significant. But the exercise shows that it is possible to derive empirical constraints on quasar ages from proximity effect signatures, which might even be enhanced by using data of higher spectral resolution.

\section{Conclusions}

We have presented new evidence of the line-of-sight proximity effect as a universal phenomenon occurring in the spectra of high-redshift quasars. Even though our spectra are limited in spectral resolution, their high $S / N$ and the power of the flux transmission method has enabled us to demonstrate the presence of the effect for every single line of sight, for the first time.

Our estimate of the mean $U V$ background intensity for the redshift range $2.7<z<4$ is $\log \left(J_{v}\right)=-21.03_{-0.22}^{+0.15}$, in very good agreement with literature values for similar redshift ranges. We made a careful assessment of the error budget using extensive Monte-Carlo simulations. The errors are clearly dominated by cosmic variance, which implies that better spectral resolution would not necessarily have a dramatic impact on the measurement accuracy. Of course, high resolution spectra would be advantageous for a more detailed analysis of several other aspects of the proximity effect, such as the effects of gravitational clustering of absorbers near the QSO (Rollinde et al. 2005), which may overestimate the value of the UV background by up to a factor of 3 (Loeb \& Eisenstein 1995).

We have quantified the strength of the proximity effect in individual spectra and find that this shows a higher dispersion than expected from only statistical shot noise errors. Among the most likely contributors for this additional dispersion are again gravitational clustering of absorbers near the QSO, or QSO variability over very long timescales; fluctuations of the UV background are also possible but unlikely to play a major role at this redshift range. We presented a speculative, but conceptually simple observational test to search for signatures of finite quasar ages in the optical depth profiles derived for a single quasar, and we even tentatively identified a candidate where such a pattern might be visible.

Acknowledgements. We would like to thank Prof. Piero Rafanelli and Dr. Stefano Ciroi for all the inspiring discussions and support. A.D.A. is grateful to the Fondazione Ing. Aldo Gini and the University of Padova for providing grants for this work. A.D.A. and G.W. acknowledge support by a HWP grant from the state of Brandenburg, Germany.

\section{References}

Bajtlik, S., Duncan, R. C., \& Ostriker, J. P. 1988, ApJ, 327, 570

Bolton, J. S., Haehnelt, M. G., Viel, M., \& Springel, V. 2005, MNRAS, 357, 1178

Cardelli, J. A., Clayton, G. C., \& Mathis, J. S. 1989, ApJ, 345, 245

Carswell, R. F., Whelan, J. A. J., Smith, M. G., Boksenberg, A., \& Tytler, D. 1982, MNRAS, 198, 91

Cooke, A. J., Espey, B., \& Carswell, R. F. 1997, MNRAS, 284, 552

Croft, R. A. C., Weinberg, D. H., Pettini, M., Hernquist, L., \& Katz, N. 1999, ApJ, 520, 1

Croom, S. M., Boyle, B. J., Shanks, T., et al. 2005, MNRAS, 356, 415

Fardal, M. A., Giroux, M. L., \& Shull, J. M. 1998, AJ, 115, 2206

Fechner, C., Baade, R., \& Reimers, D. 2004, A\&A, 418, 857

Gaskell, C. M. 1982, ApJ, 263, 79

Giallongo, E., Christiani, S., D’Odorico, S., Fontana, A., \& Savaglio, S. 1996, ApJ, 466, 46

Haardt, F., \& Madau, P. 1996, ApJ, 461, 20

Hawkins, M. R. S., \& Veron, P. 1993, MNRAS, 260, 202

Hewett, P. C., Foltz, C. B., \& Chaffee, F. H. 1995, AJ, 109, 1498

Hopkins, P. F., Hernquist, L., Cox, T. J., et al. 2006, ApJS, 163, 1

Hu, E. M., Kim, T.-S., Cowie, L. L., Songaila, A., \& Rauch, M. 1995, AJ, 110, 1526

Hui, L., \& Rutledge, R. E. 1999, ApJ, 517, 541

Kim, T.-S., Cristiani, S., \& D’Odorico, S. 2001, A\&A, 373, 757

Kim, T.-S., Carswell, R. F., Cristiani, S., D’Odorico, S., \& Giallongo, E. 2002, MNRAS, 335, 555

Liske, J., \& Williger, G. M. 2001, MNRAS, 328, 653

Loeb, A., \& Eisenstein, D. J. 1995, ApJ, 448, 17

Lopez, S., Maza, J., Masegosa, J., \& Marquez, I. 2001, A\&A, 366, 387

Lopez, S., Reimers, D., D’Odorico, S., \& Prochaska, J. X. 2002, A\&A, 385, 778

Lu, L., Sargent, W. L. W., Womble, D. S., \& Takada-Hidai, M. 1996, ApJ, 472, 509

McDonald, P., Seljak, U., Cen, R., et al. 2005, ApJ, 635, 761

Morton, D. C. 2003, ApJS, 149, 205

Murdoch, H. S., Hunstead, R. W., Pettini, M., \& Blades, J. C. 1986, ApJ, 309, 19

Osmer, P. S., Porter, A. C., \& Green, R. F. 1994, ApJ, 436, 678

Peacock, J. A. 1999, Cosmological Physics (Cambridge University Press)

Pentericci, L., Fan, X., Rix, H.-W., et al. 2002, AJ, 123, 2151

Petitjean, P., Webb, J. K., Rauch, M., Carswell, R. F., \& Lanzetta, K. 1993, MNRAS, 262, 499

Pettini, M., Smith, L. J., King, D. L., \& Hunstead, R. W. 1997, ApJ, 486, 665

Rauch, M., Miralda-Escude, J., Sargent, W. L. W., et al. 1997, ApJ, 489, 7

Reimers, D., Köhler, S., Wisotzki, L., et al. 1997, A\&A, 327, 890

Rollinde, E., Srianand, R., Theuns, T., Petitjean, P., \& Chand, H. 2005, MNRAS, 361, 1015

Savaglio, S., Christiani, S., D’Odorico, S., et al. 1997, A\&A, 318, 347

Schaye, J., Aguirre, A., Kim, T.-S., et al. 2003, ApJ, 596, 768

Schlegel, D. J., Finkbeiner, D. P., \& Davis, M. 1998, ApJ, 500, 525

Schneider, D. P., Schmidt, M., \& Gunn, J. E. 1991, AJ, 101, 2004

Scott, J., Bechtold, J., Dobrzycki, A., \& Kulkarni, V. P. 2000, ApJ, 130, 67

Steidel, C. C. 1990, ApJS, 72, 1

Storrie-Lombardi, L. J., \& Wolfe, A. M. 2000, ApJ, 543, 552

Suzuki, N. 2006, ApJS, 163, 110

Suzuki, N., Tytler, D., Kirkman, D., O’Meara, J. M., \& Lubin, D. 2005, ApJ, 618,592

Tytler, D., \& Fan, X.-M. 1992, ApJS, 79, 1

Vanden Berk, D. E., Richards, G. T., Bauer, A., et al. 2001, AJ, 122, 549

Véron-Cetty, M.-P., \& Véron, P. 2006, A\&A, 455, 773

Williger, G. M., Baldwin, J. A., Carswell, R. F., et al. 1994, ApJ, 428, 574

Worseck, G., \& Wisotzki, L. 2006, A\&A, 450, 495

Worseck, G., Fechner, C., Wisotzki, L., \& Dall'Aglio, A. 2007, A\&A, 473, 805

Young, P. J., Sargent, W. L. W., Boksenberg, A., Carswell, R. F., \& Whelan,

J. A. J. 1979, ApJ, 229, 891

Yu, Q., \& Tremaine, S. 2002, MNRAS, 335, 965

Zhang, Y., Anninos, P., \& Norman, M. L. 1995, ApJ, 453, L57

Zuo, L. 1993, A\&A, 278, 343 
A. Dall'Aglio et al.: The line-of-sight proximity effect in individual quasar spectra, Online Material p 1

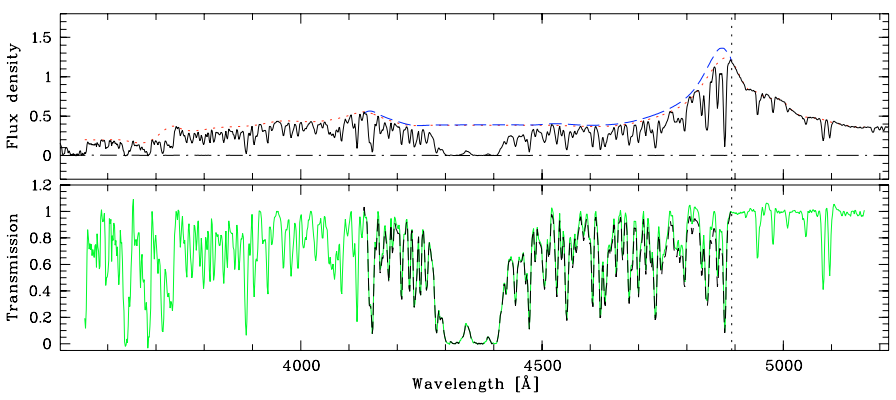

Fig. A.1. CTQ 0247.

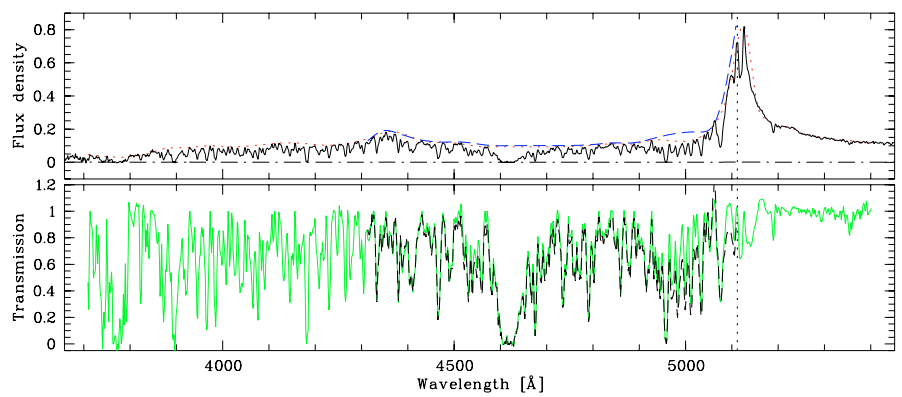

Fig. A.2. CTQ 1005.

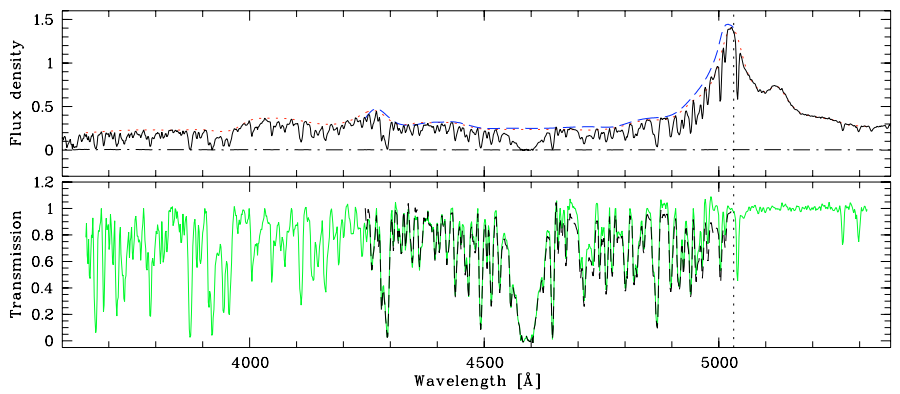

Fig. A.3. CTQ 0460.

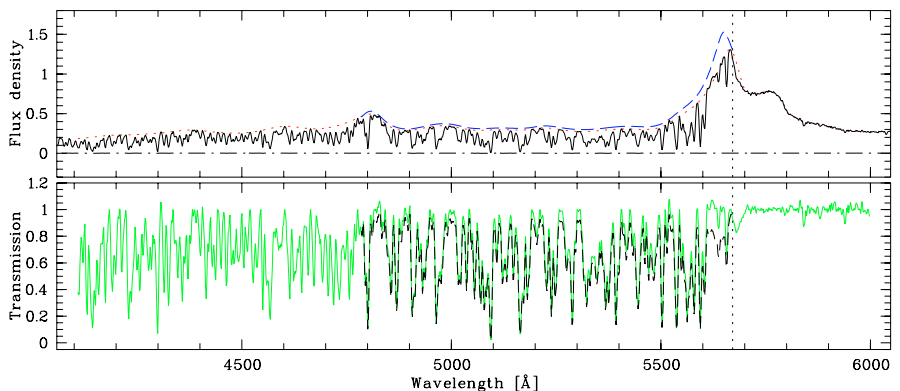

Fig. A.4. H 0055-2659.

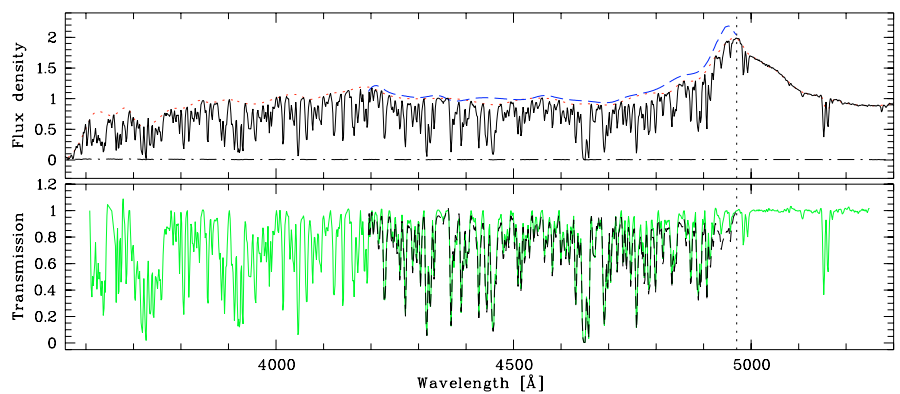

Fig. A.5. HE 0940-1050.

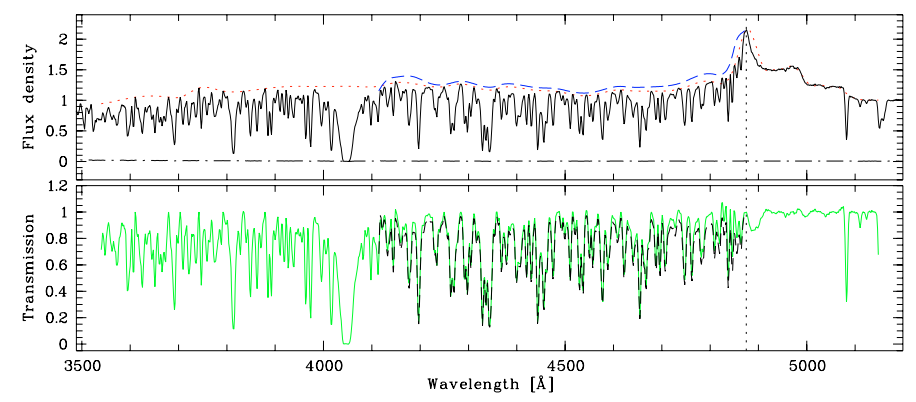

Fig. A.6. HE 2243-6031.

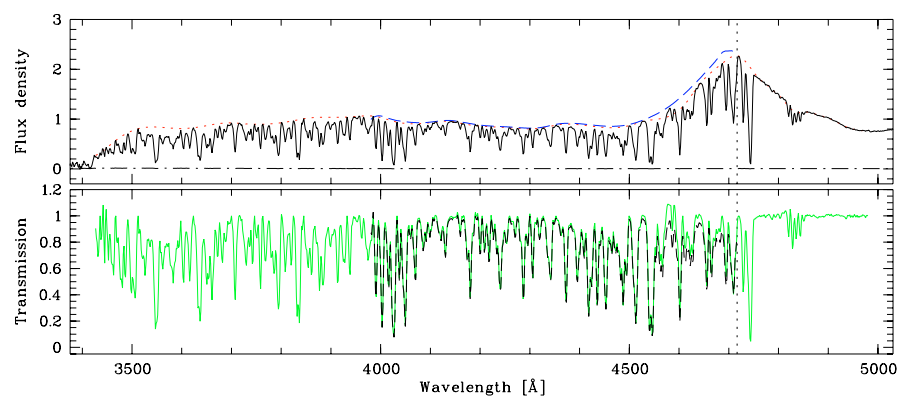

Fig. A.7. HE 2347-4342.

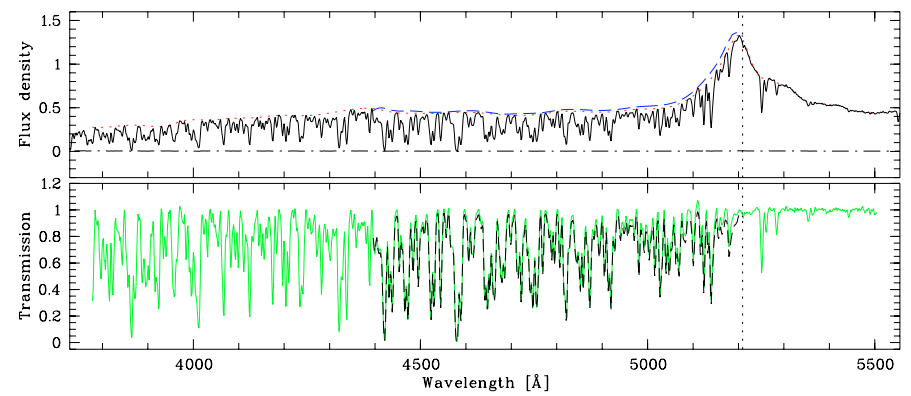

Fig. A.8. PKS 2126-15.

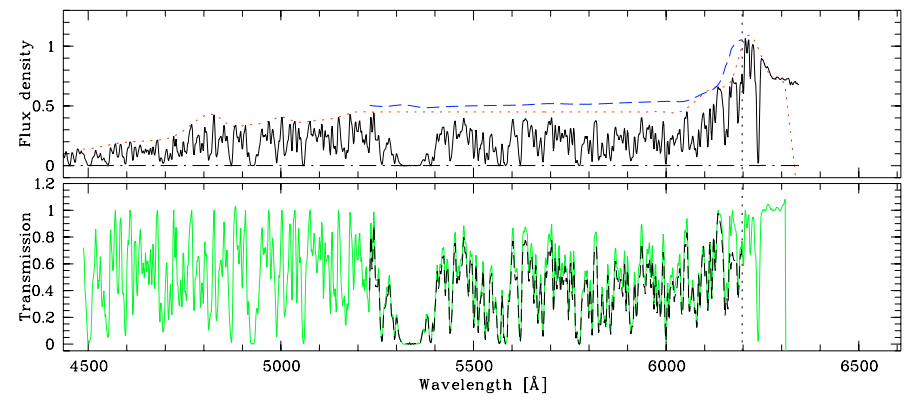

Fig. A.9. Q 0000-26.

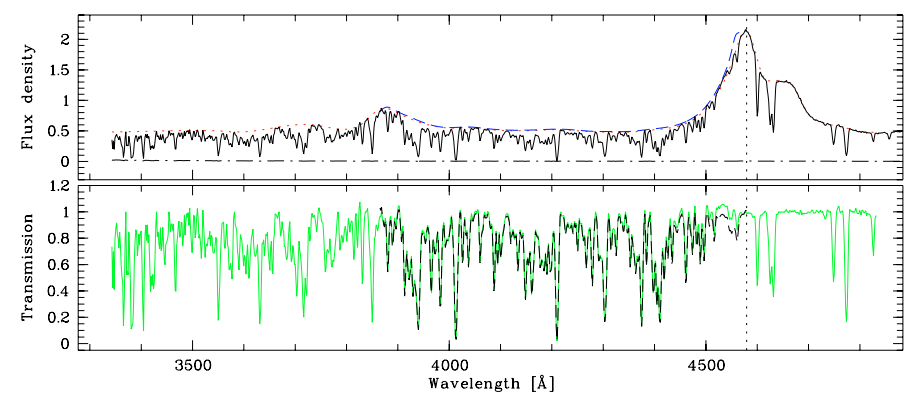

Fig. A.10. Q 0002-422. 
A. Dall'Aglio et al.: The line-of-sight proximity effect in individual quasar spectra, Online Material p 2

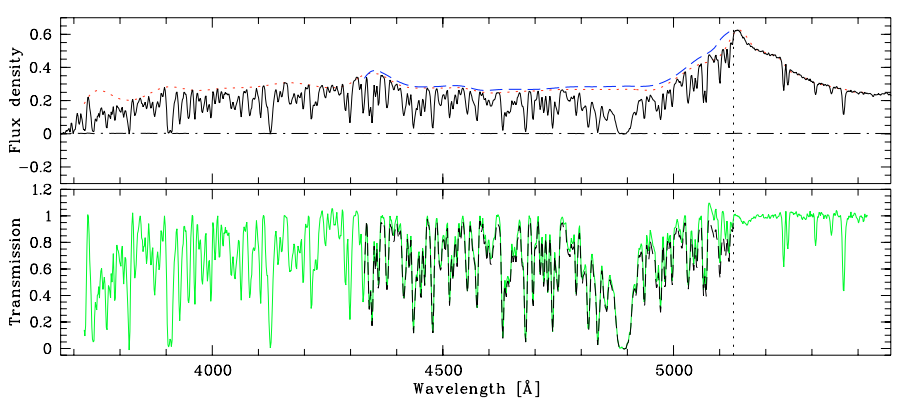

Fig. A.11. Q 0347-383.

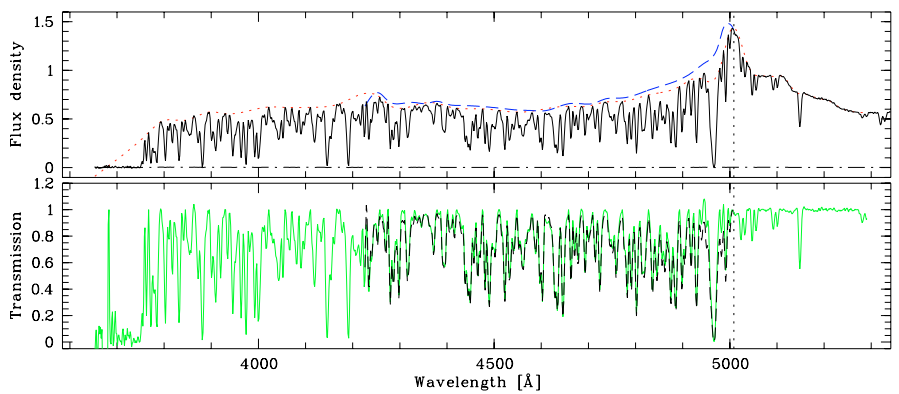

Fig. A.12. Q 0420-388.

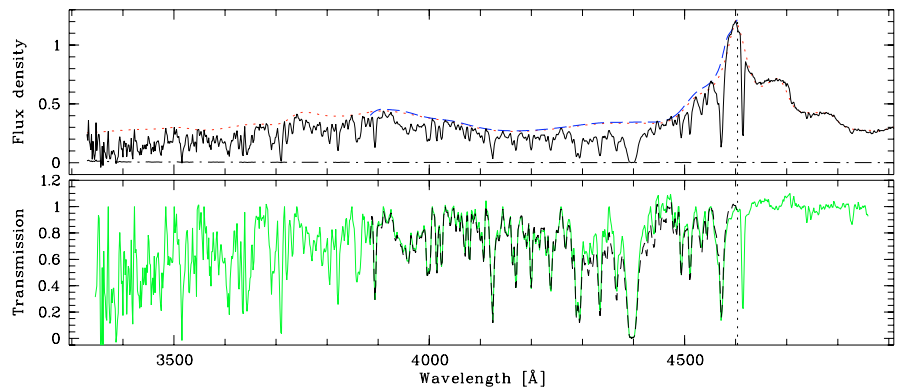

Fig. A.13. Q 0913+0715.

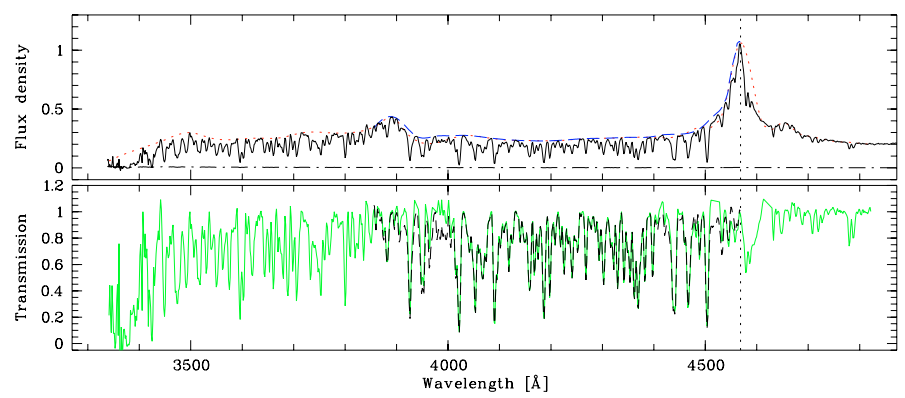

Fig. A.14. Q 1151+0651.

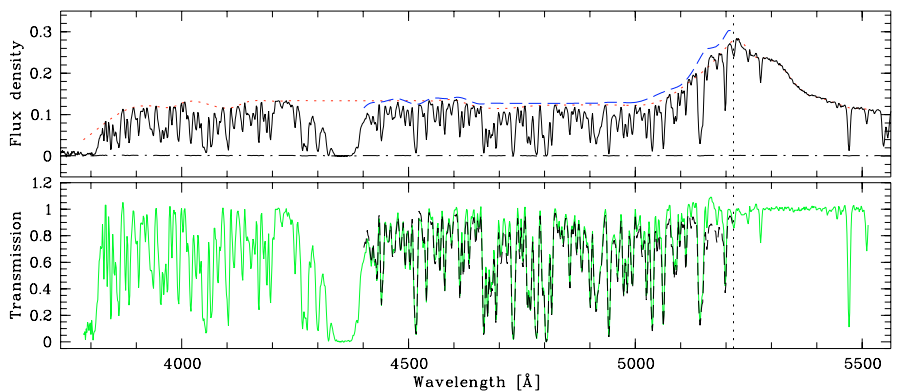

Fig. A.15. Q 1209+0919.

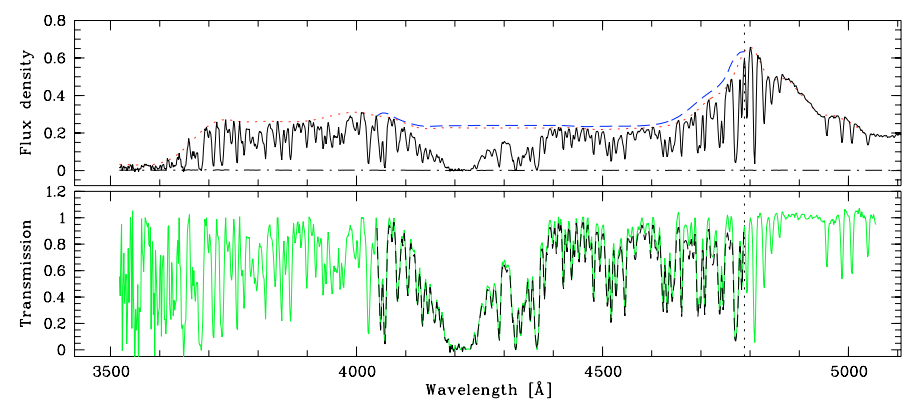

Fig. A.16. Q 1223+1753.

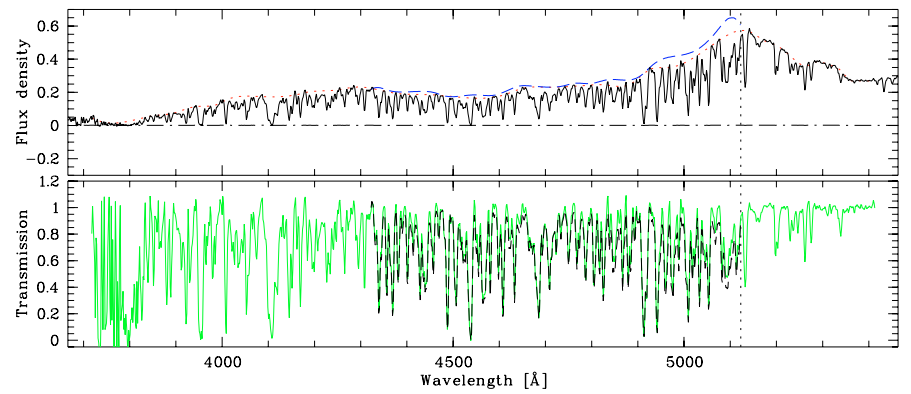

Fig. A.17. Q 2139-4434. 